\title{
Escola postural: revisão sistemática dos programas desenvolvidos para escolares no Brasil
}

\author{
Matias Noll \\ Cláudia Tarragô Candotti* \\ Adriane Vieira ${ }^{* * *}$
}

\begin{abstract}
Resumo: Este estudo tem como objetivo apresentar e discutir os programas de Escola Postural desenvolvidos para escolares no Brasil. Foi utilizada uma metodologia de revisão sistemática de artigos científicos em várias bases de dados e no Banco de Teses e Dissertações da Capes. A partir das nove publicações incluídas neste estudo concluiu-se que os programas de Escola Postural contribuem para a aquisição de conhecimentos teóricos e de hábitos posturais saudáveis durante a execução das Atividades de Vida Diárias. No entanto, estudos que visem identificar se os conhecimentos e os hábitos posturais adquiridos são mantidos no transcorrer do tempo ainda são escassos.
\end{abstract}

Palavras-chave: Postura. Coluna Vertebral. Educação em Saúde. Estudantes. Brasil.

\section{INTRODUÇÃo}

Atualmente, as ocorrências de dor nas costas e de alterações posturais estão entre os maiores problemas socioeconômicos e de saúde pública enfrentados pelos países em desenvolvimento, pois contribuem consideravelmente para limitar a vida ativa dos trabalhadores (SOUZA, 1996; SILVA; MENEZES; NOAL, 2009; CANDOTTI; NOLL; CRUZ, 2010), resultando em prejuízos em função do tempo de afastamento do trabalho e aposentadorias

\footnotetext{
'Mestre em Ciências do Movimento Humano pelo Programa de Pós-Graduação em Ciências do Movimento Humano - ESEF/UFRGS. E-mail: matiasnoll@yahoo.com.br

"Professora do curso de Fisioterapia e do Programa de Pós-Graduação (Mestrado e Doutorado) da Universidade Federal do Rio Grande do Sul.E-mail: claudia.candotti@ufrgs.br

".*Professora do curso de Fisioterapia e do Programa de Pós-Graduação (Mestrado e Doutorado) da Universidade Federal do Rio Grande do Sul. E-mail: adriane.vieira@gmail.com
} 
precoces (CARDON; CLERCQ; BOURDEAUDHUIJ, 2000). As altas despesas resultantes do diagnóstico e tratamento; os prejuízos gerados às instituições, tanto públicas quanto privadas; e a falta de resultados das práticas terapêuticas convencionais, deram origem à "Back School" (ANDRADE; ARAUJO; VILAR, 2005).

A "Back School", que consiste em um programa de educação e treinamento postural utilizado na prevenção e no tratamento de indivíduos com dores nas costas (ANDRADE; ARAUJO; VILAR, 2005), pode, dependendo da origem dos pesquisadores e de sua concepção epistemológica, ser também denominada de "Health Education, Postural Program, Postural School, Back Care Education", entre outros. Quando o termo original "Back School" é traduzido para o português, o mesmo também recebe distintas nomenclaturas, sendo as mais utilizadas: Escola de Coluna (CHUNG, 1996; ANDRADE; ARAUJO; VILAR, 2005), Escola de Postura (KNOPLICH, 2006; FERREIRA; NAVEGA, 2010), Escola Postural (SOUZA; VIEIRA, 2003, UES; MORAES, 2003), e Programa de Educação Postural (BENINI; KAROLCZAK, 2010; CANDOTTI et al, 2011). Neste artigo adotou-se como a tradução mais adequada de "Back School", a expressão "Escola Postural". Não obstante, ao longo desta revisão será respeitada a nomenclatura adotada pelos diferentes autores.

Tem sido referenciado que participantes de "Escolas Posturais", de diferentes faixas etárias, tendem a modificar positivamente sua postura durante as Atividades de Vida Diária (AVD's), bem como seus conhecimentos teóricos sobre a coluna vertebral (ROBERTSON; LEE, 1990, CARDON; CLERCQ; BOURDEAUDHUIJ, 2002, MÉNDEZ; GÓMEZ-CONESA, 2010). Desse modo, as Escolas Posturais podem ser oferecidas a públicos distintos, como, por exemplo, terceira idade, crianças e adolescentes (SPENCE; JENSEN; SHEPARD, 1984, ROBERTSON; LEE, 1990, CARDON; CLERCQ; BOURDEAUDHUIJ, 2002, CANDOTTI et al, 2009; REBOLHO; CASAROTTO; AMADO, 2009, MÉNDEZ; GÓMEZ-CONESA, 2010), desde que seus conteúdos teóricos e práticos sejam adaptados às diferentes necessidades. 
O sucesso deste método preventivo e de tratamento fez com que, nas últimas décadas, em diversos locais do mundo, as "Escolas Posturais" fossem uma opção eficaz para atenuar as altas prevalências de alterações posturais e de dores na coluna vertebral (SOUZA; VIEIRA, 2003, FERREIRA; NAVEGA, 2010). Especificamente no Brasil, o ensino das AVD's por meio de "Escolas Posturais" e metodologias similares tem sido amplamente pesquisada como um recurso para o tratamento de dores nas costas na população de adultos (CHUNG, 1996, VIEIRA; SOUZA, 2002, SOUZA; VIEIRA, 2003, UES; MORAES, 2003, FERREIRA; NAVEGA, 2010, TOBO et al, 2010, BORGES et al, 2011). Esses programas apresentam diferentes estruturas em termos de números de aula, tempo de duração de cada aula, conteúdos abordados. Entretanto, observa-se que todos os programas contemplam tanto conteúdos teóricos, como a anatomia e fisiologia da coluna vertebral e a importância dos exercícios físicos e cuidados com a postura durante a realização de AVD's para evitar dores musculoesqueléticas, quanto atividades práticas, como vivências de posturas adequadas e exercícios. Os programas de Escola Postural têm sido oferecidos a grupos de adultos que apresentam dores nas costas em diferentes contextos, como, por exemplo, em Unidades Básicas de Saúde (UES; MORAES, 2003, FERREIRA; NAVEGA, 2010, BORGES et al, 2011), projetos de extensão universitários oferecidos à comunidade (VIEIRA; SOUZA, 2002, SOUZA; VIEIRA, 2003), hospitais/ clínicas universitárias (CHUNG, 1996; TOBO et al, 2010).

No entanto, a ocorrência de dor nas costas e as alterações posturais, além de estarem presentes em larga escala em adultos, manifestam-se também em grandes proporções na infância e na adolescência (DETSCH; CANDOTTI, 2001, LIMON; VALINSKY; SHALOM, 2004, SHEHAB; JARALLAH, 2005, SKOFFER, 2007). Paananen et al (2010) demonstraram que a ocorrência de dor musculoesquelética em duas ou mais áreas anatômicas é muito frequente em jovens escolares. Do mesmo modo, outros estudos, em que avaliaram escolares das cidades de Jaguariúna - SP (SANTOS et al, 2009) e de São Leopoldo - RS (DETSCH et al, 2007), verificaram uma alta prevalência de alterações posturais laterais e 
ântero-posteriores, acometendo mais de $66 \%$ dos avaliados. Especula-se que a dor nas costas e os problemas posturais em jovens podem ter causa multifatorial (SIIVOLA et al, 2004), como: utilizar mochilas pesadas e transportá-las de modo assimétrico (KELLIS; EMMANOUILIDOU, 2010), permanecer longos períodos sentado, utilizar cadeiras e mesas que não possuam dimensões adequadas ao tamanho do usuário (LIMON; VALINSKY, 2004), assumir posturas que não mantenham as curvaturas fisiológicas da coluna vertebral durante a execução de AVD's (VANDERTHOMMEN et al, 1999; WOMERSLEY; MAY, 2006, TREVELYAN; LEGG, 2006), utilizar calçados de salto alto que gerem compensações na busca do equilíbrio postural (PEZZAN et al, 2011), dormir menos de sete horas por dia, estar com sobrepeso (PAANANEN et al, 2010; AUVINEN et al, 2010) e apresentar algum fator psicossocial, como, por exemplo, depressão e ansiedade (LIMON; VALINSKY, 2004).

Desta forma, sabendo-se que a postura corporal executada durante as AVD's está presente entre os fatores de risco associados à lombalgia e à ocorrência de problemas posturais na população de escolares (MÉNDEZ; GÓMEZ-CONESA, 2010), especula-se que a mesma tem importantes implicações para a saúde e o bem-estar do ser humano (CANDOTTI; NOLL; CRUZ, 2010). Isso porque ela determina a quantidade e a distribuição do esforço sobre os vários ossos, músculos, tendões, ligamentos e discos intervertebrais, podendo potencializar ou amenizar os malefícios e sobrecargas resultantes na coluna vertebral (KARAHAN; BAYRAKTAR, 2004).

Neste contexto, torna-se relevante a inclusão de propostas educativas e preventivas nas escolas que visem à diminuição das altas prevalências de alterações posturais e de dores na coluna vertebral em crianças e adolescentes (FERNANDES; CASAROTTO; JOÃO, 2008). As "Escolas Posturais" apresentam estratégias pedagógicas direcionadas ao conhecimento e aquisição de hábitos posturais saudáveis que visam prevenir e minimizar a dor e as alterações posturais. Seus conteúdos e métodos de ensino podem ser adaptados as diferentes faixas de desenvolvimento 
cognitivo e motor, adequando-se à infância e à adolescência.

Um passo inicial para que o desenvolvimento de "Escolas Posturais" torne-se uma realidade no cotidiano escolar é conhecer os programas existentes, seus métodos de ensino e avaliação, bem como seus resultados. Deste modo, o presente estudo de revisão sistemática objetivou apresentar e discutir os programas de "Escola Postural" desenvolvidos nas últimas quatro décadas para a população de escolares no Brasil, e apontar direções e perspectivas para o desenvolvimento de estudos e intervenções no meio escolar.

\section{Metodologia}

Para cumprir com o propósito deste estudo, foi utilizada a metodologia de revisão sistemática de artigos científicos, a qual consiste em uma forma de síntese dos resultados de pesquisas encontradas em várias bases de dados relacionados a um problema específico (GALVÃO; SAWADA; TREVIZAN, 2004). As bases de dados consultadas no presente estudo foram: Scopus, Science Direct, PubMed, Scielo e o Banco de Teses e Dissertações da Capes (BTDC). As palavras-chave utilizadas na busca pelos estudos foram: Postural Program; Postural School; Back School; Back Care Education; Back Education; e os respectivos termos em português. Os estudos encontrados, para integrar a presente revisão sistemática, deveriam preencher os seguintes critérios: (a) desenvolver um programa teórico e/ou prático educativo e/ou preventivo relacionado ao ensino da execução adequada das AVD's e assuntos relacionados à postura corporal e coluna vertebral; (b) terem sido desenvolvidos a partir da década de 1970 no Brasil; e (c) que a população alvo fosse crianças e/ou adolescentes. Optou-se pela inclusão de publicações a partir de 1970, pois esta data caracteriza a origem do desenvolvimento das "Escolas Posturais" (FORSSEL, 1980; FORSSEL, 1981, ANDRADE; ARAUJO; VILAR, 2005).

A seleção inicial ocorreu pela simples leitura dos títulos 
encontrados, sendo descartados aqueles não relacionados às palavras-chave definidas. Para os estudos potencialmente elegíveis, além da leitura dos títulos foram também lidos os resumos. Os artigos que aparentemente cumpriram com os critérios de inclusão foram lidos e analisados na íntegra, sendo finalmente incluídos aqueles que contemplassem todos os três critérios estabelecidos na presente revisão sistemática. Adicionalmente, as referências bibliográficas de cada publicação foram examinadas objetivando encontrar artigos ainda não rastreados pela busca eletrônica.

A Figura 1 apresenta o fluxograma da estratégia adotada para busca, realizada nos meses de outubro e novembro de 2011, e inclusão dos artigos e as razões de exclusão para os artigos não inseridos. Dos 291 artigos inicialmente identificados a partir das palavras-chave, 249 foram excluídos, pois não foram desenvolvidos a partir da década de 70 no Brasil. Dos 42 artigos restantes, ao serem analisados na íntegra, 36 foram excluídos por não abrangerem a população de escolares. Assim, somente 6 artigos cumpriram com os todos os critérios de inclusão da revisão. Ainda foram incluídos 2 artigos obtidos por meio da análise das referências bibliográficas e uma dissertação de mestrado obtida no BTDC. 
Figura 1 - Fluxograma da seleção de artigos

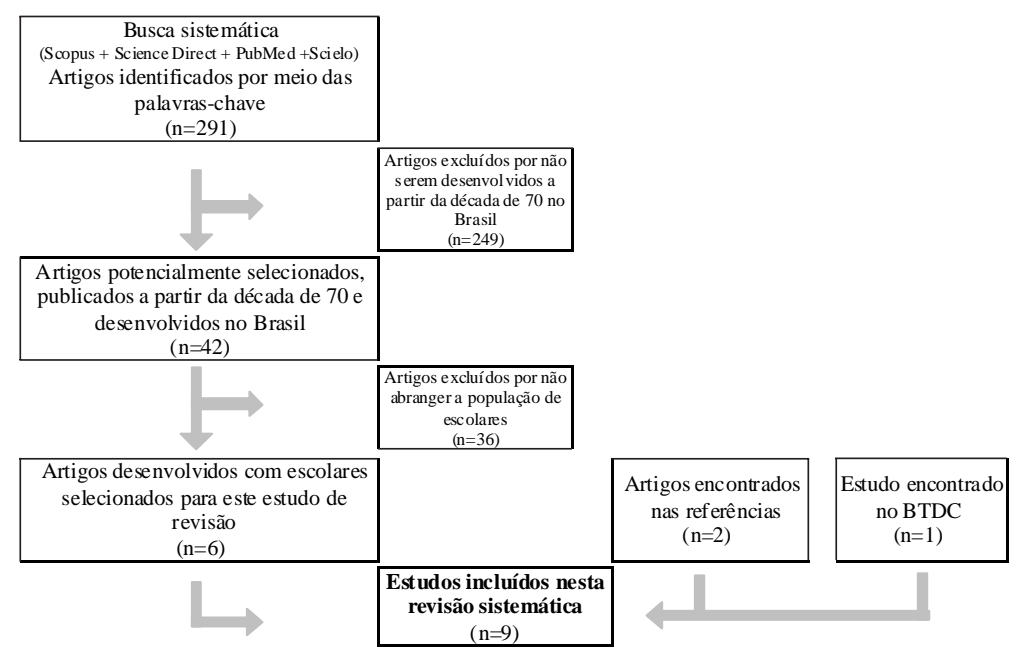

Fonte: autores

\section{Programas de escola postural desenvolvidos para a POPULAÇÃO DE ESCOLARES NO BRASIL}

No Quadro 1 são apresentados e descritos os nove estudos selecionados e incluídos nesta revisão sistemática. A seguir, listarse-ão os artigos por ordem cronológica, assim como uma breve discussão e reflexão sobre cada um deles e suas principais características. Sabendo-se que os programas de ensino da postura adequada nas AVD's apresentam nomenclaturas distintas, optou-se por manter as nomenclaturas descritas originalmente na publicação destes estudos. São discutidos aspectos relacionados à metodologia de ensino (técnicas de ensino, periodicidade e duração do programa de ensino), instrumentos de coleta de dados e resultados do programa de ensino. 


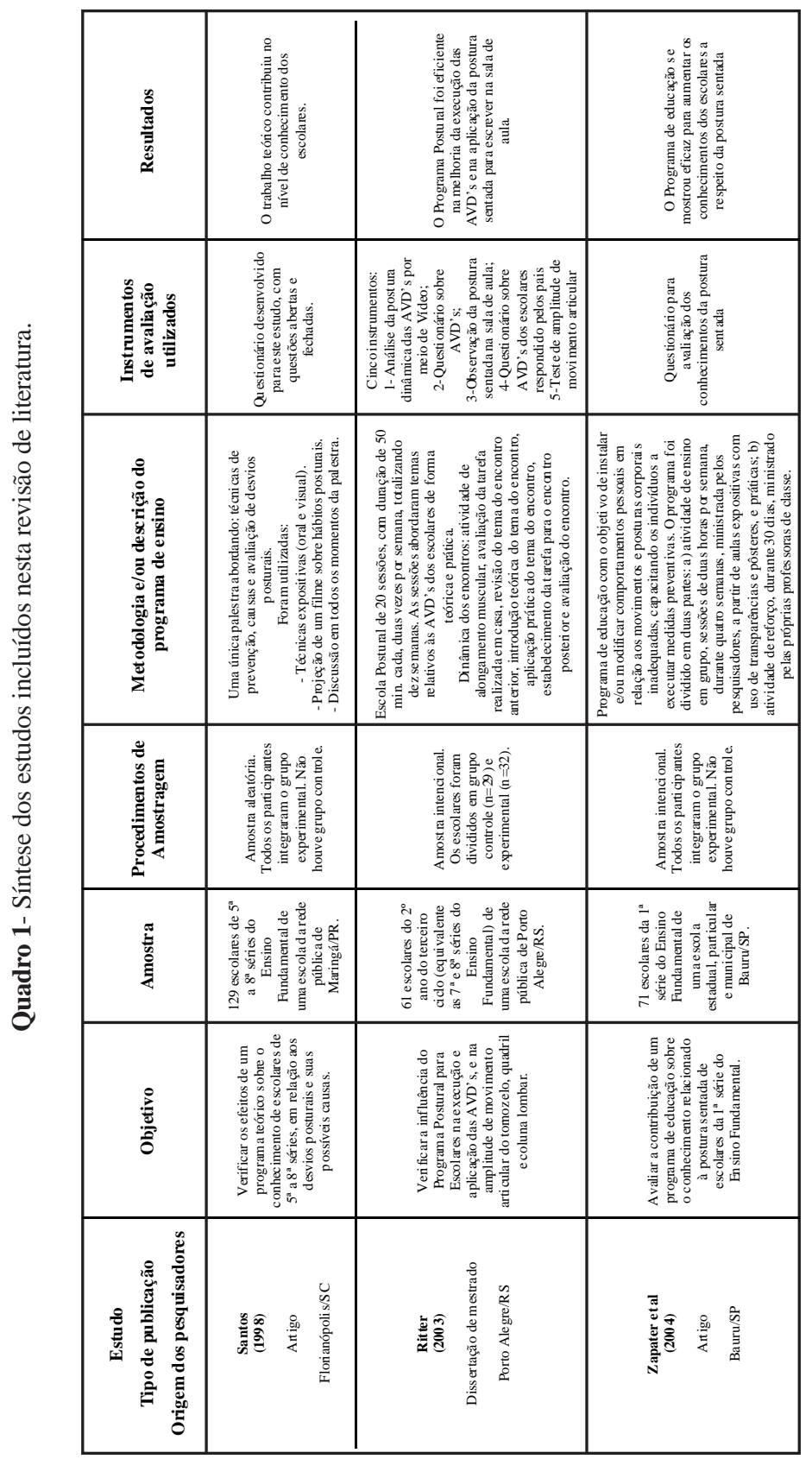

Movimento, Porto Alegre, v. 18, n. 04, p. 265-291, out/dez de 2012. 
Escola postural: revisão sistemática dos ...

\begin{tabular}{|c|c|c|c|}
\hline 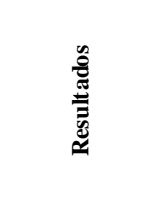 & 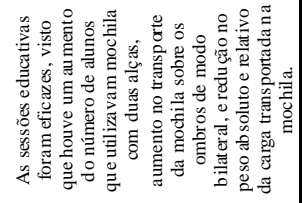 & 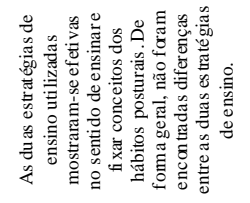 & 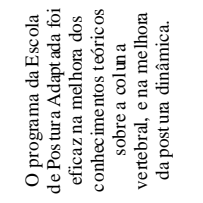 \\
\hline 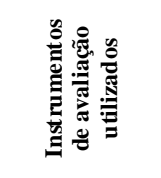 & 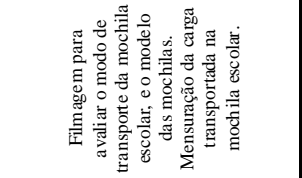 & 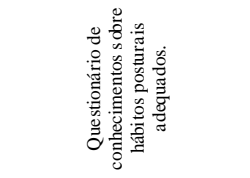 & 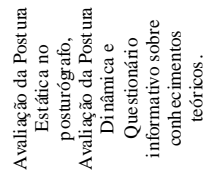 \\
\hline 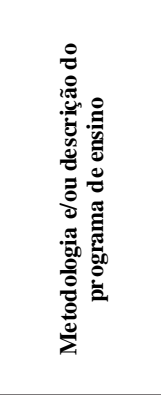 & 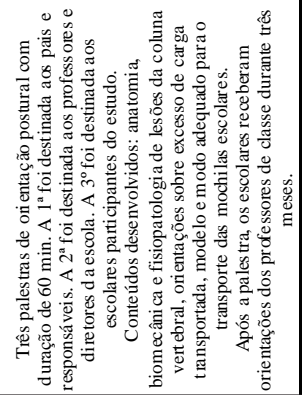 & 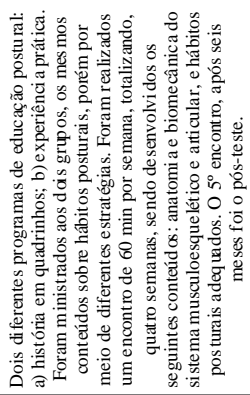 & 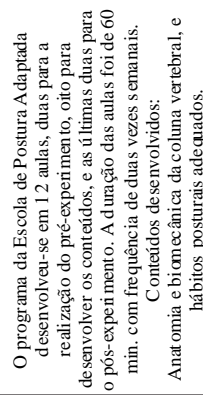 \\
\hline 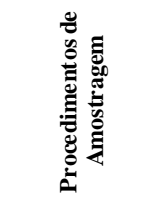 & 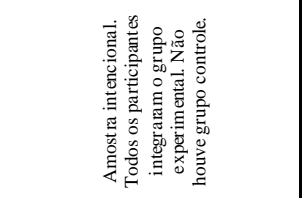 & 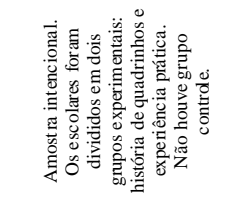 & 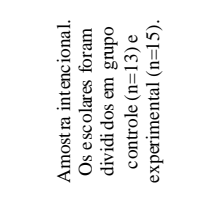 \\
\hline 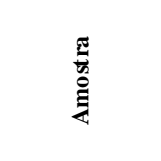 & 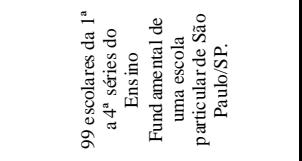 & 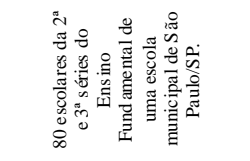 & 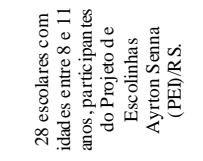 \\
\hline$\frac{\stackrel{2}{a}}{\stackrel{0}{0}}$ & 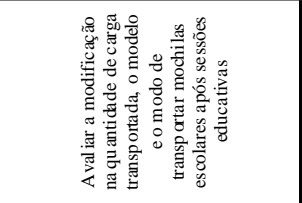 & 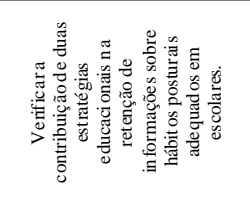 & 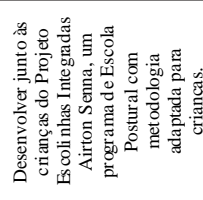 \\
\hline 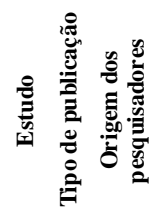 & 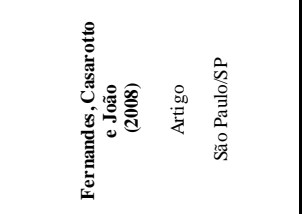 & 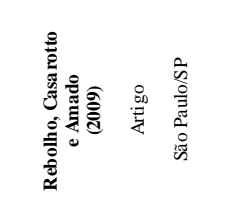 & 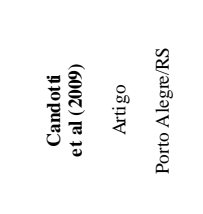 \\
\hline
\end{tabular}

Vovimento, Porto Alegre, v. 18, n. 04, p. 265-291, out/dez de 2012. 


\begin{tabular}{|c|c|c|c|}
\hline 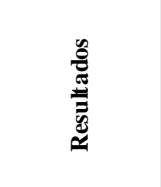 & 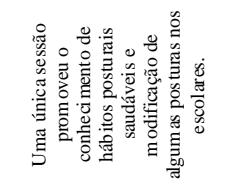 & 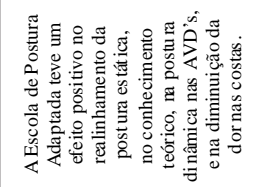 & 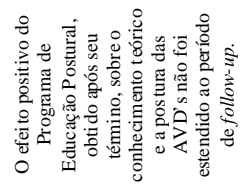 \\
\hline 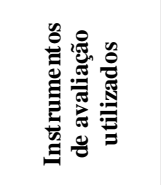 & 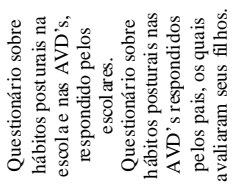 & 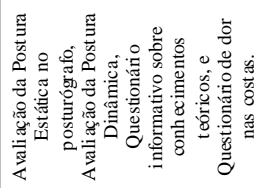 & 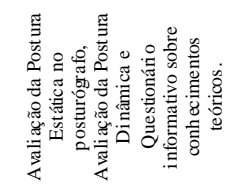 \\
\hline 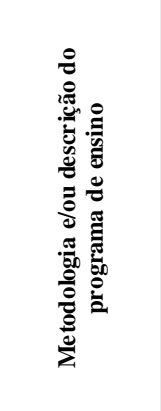 & 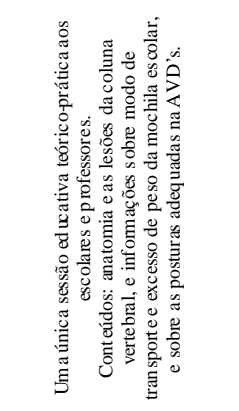 & 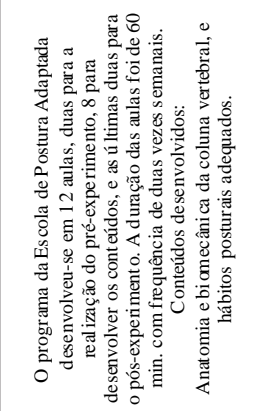 & 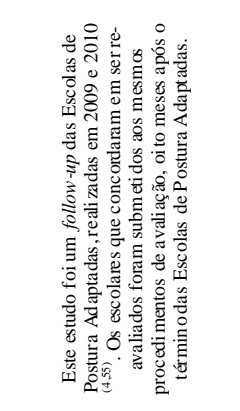 \\
\hline 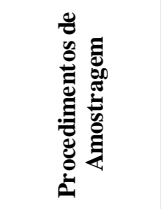 & 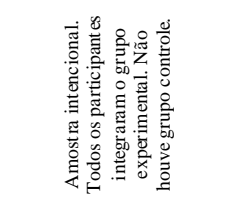 & 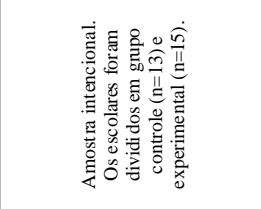 & 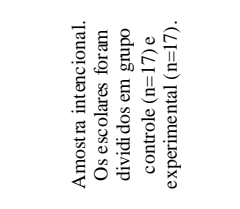 \\
\hline 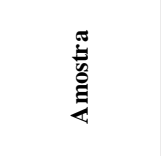 & 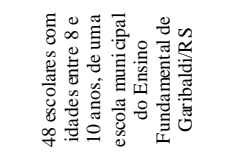 & 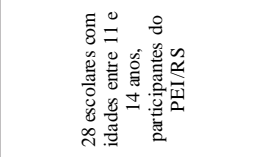 & 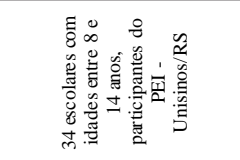 \\
\hline : & 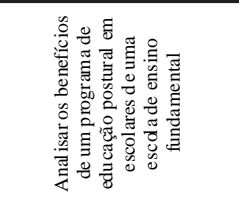 & 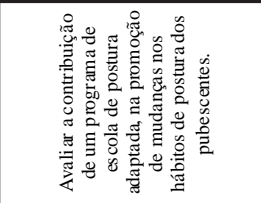 & 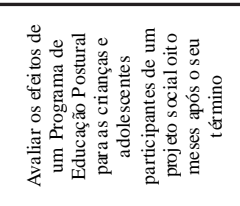 \\
\hline 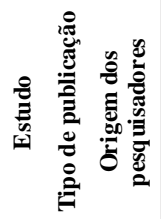 & 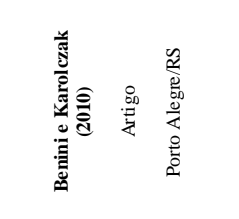 & 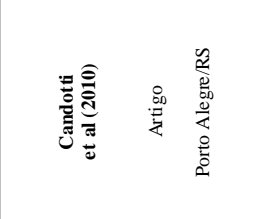 & 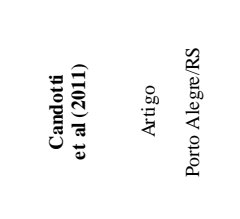 \\
\hline
\end{tabular}

Movimento, Porto Alegre, v. 18, n. 04, p. 265-291, out/dez de 2012. 
O primeiro estudo sobre um programa de ensino de AVD's para escolares, realizado no Brasil, foi desenvolvido por Santos (1998), na década de 90. Este estudo teve como objetivo central verificar os efeitos de um programa teórico sobre o conhecimento de escolares de $5^{\mathrm{a}}$ a $8^{\mathrm{a}}$ séries, em relação aos desvios posturais e suas possíveis causas. O programa de ensino foi composto por uma única palestra, a qual foi realizada por acadêmicos, abordando os seguintes conteúdos: técnicas de prevenção, causas e avaliação de desvios posturais. Foram utilizadas, durante a palestra, técnicas expositivas (oral e visual), momentos de discussão ao longo das atividades, e foi projetado um filme sobre os hábitos posturais adequados nas AVD's. Santos (1998) concluiu, a partir da utilização de questionário aplicado após um período de 40 dias da intervenção, que o seu programa de Educação Postural foi eficaz na melhora do nível de conhecimento dos escolares. No entanto, o autor afirma que não se pode garantir que com apenas esta contribuição do programa de ensino realizado na melhora do conhecimento dos escolares, ocorra uma mudança dos hábitos posturais nas AVD's.

Cronologicamente, o próximo estudo desenvolvido no Brasil foi realizado por Ritter (2003) o qual desenvolveu uma Escola Postural de 20 sessões. As sessões abordaram temas relativos à evolução do homem e a coluna vertebral, estrutura e função da coluna vertebral, e postura adequadas nas AVD's, de forma teórica e prática, como por exemplo, postura ao pegar objeto do solo, postura ao transportar o material escolar e postura sentada. Todos os encontros seguiram a seguinte dinâmica: atividade de alongamento muscular, avaliação da tarefa realizada em casa, revisão do tema da aula anterior, introdução teórica e aplicação prática do tema do encontro, estabelecimento da tarefa para o encontro posterior e avaliação do encontro. Foram utilizados diversos materiais, tais como, esqueleto humano, materiais esportivos e recicláveis, e diversos recursos de aprendizagem, dentre eles, momentos de discussões e atividades recreativas.

Ritter (2003) utilizou cinco instrumentos avaliativos: (a) Análise da postura dinâmica das AVD's através de vídeo, protocolo 
proposto por Rocha e Souza (1999); (b) Questionário sobre AVD's, validado em estudo piloto, o qual objetivou verificar a forma como os escolares executam as AVD's; (c) Observação da postura sentada para escrever em sala de aula, por meio de filmagens; (d) Questionário sobre as AVD's dos escolares respondido pelos pais, cujo objetivo era de que os mesmos avaliassem os seus filhos nas atividades domésticas; e (e) Teste de amplitude de movimento das articulações do tornozelo, quadril, e da coluna lombar. Os resultados encontrados por Ritter (2003) demonstraram que os participantes da Escola Postural apresentaram melhora significativa em todas as AVD's avaliadas através de vídeo, e na observação da tarefa de sentar para escrever em sala de aula por meio de filmagens. No entanto, o mesmo não foi observado no questionário sobre as AVD's, na observação das tarefas investigadas no ambiente doméstico e na amplitude de movimento articular, sugerindo que este programa não contribuiu para a modificação destas variáveis.

Um ano mais tarde, Zapater et al (2004) publicaram um estudo cujo objetivo foi avaliar a contribuição de um programa de educação sobre o conhecimento relacionado à postura sentada. $\mathrm{O}$ programa de educação teve como objetivo instalar e/ou modificar comportamentos pessoais em relação aos movimentos e posturas corporais inadequadas, capacitando os indivíduos a executar medidas preventivas. O programa foi dividido em duas partes: a) a primeira parte foi ministrada pelos pesquisadores, caracterizada como atividades de ensino em grupo, duas horas semanais, ao longo de quatro semanas, com sessões expositivas, sendo utilizadas transparências e pôsteres, com o intuito de informar sobre o "que fazer", e sessões práticas, com o intuito de informar sobre "como fazer"; b) a segunda parte foi ministrada pelas próprias professoras de classe, caracterizada como atividades de reforço, durante 30 dias. Os temas das aulas, tanto da primeira quanto da segunda parte do programa, foram: anatomia básica da coluna vertebral, membro superior e inferior; efeitos e sobrecargas relacionadas à postura sentada; e como prevenir os malefícios relacionados à postura sentada. 
Foi verificado pelos autores (ZAPATER et al, 2004), por meio de um questionário aplicado logo após a finalização da intervenção, que o programa de educação se mostrou eficaz para aumentar os conhecimentos dos escolares a respeito da postura sentada. Os autores relatam a necessidade de se dar continuidade a essa linha de pesquisa, com estudos que investiguem o comportamento preventivo em longo prazo, com consequente mudança de hábito postural, além de programas preventivos que orientem o professor a identificar fatores de risco e atuar no ambiente escolar.

Nos últimos quatro anos houve um aumento no interesse dos pesquisadores em desenvolver programas educativos com a população de escolares (FERNANDES; CASAROTTO; JOÃO, 2008, REBOLHO; CASAROTTO; AMADO, 2009, CANDOTTI et al, 2009, BENINI; KAROLCZAK, 2010, CANDOTTI et al, 2010; CANDOTTI et al, 2011), os quais são descritos a seguir.

Fernandes, Casarotto e João (2008) objetivaram avaliar o efeito de sessões educativas teóricas sobre as posturas adotadas pelos escolares no transporte de mochilas escolares, sobre o modelo e modo de transporte de mochilas, e sobre a quantidade de carga transportada. Os alunos, sem aviso prévio, foram filmados no momento de chegada na escola, a partir de uma câmera de vídeo fixada discretamente na portaria principal da escola. Os dados das filmagens foram categorizados seguindo quatro modos de transporte (ombro unilateral, ombro bilateral, mãos e mãos no modelo carrinho) e três tipos de mochila (uma alça, duas alças e carrinho). Em seguida, a quantidade de carga $(\mathrm{kg})$ das mochilas foi verificada.

O programa educativo foi composto por três palestras sobre orientação postural destinadas (1) aos pais e/ou responsáveis, (2) aos professores e diretor da escola, e (3) aos escolares participantes do estudo. Nas palestras, com duração de 60 minutos, foram desenvolvidos os seguintes conteúdos: conceitos de anatomia, biomecânica e fisiopatologia das lesões de coluna vertebral; orientações sobre excesso de carga transportada, modelo e modo adequado para o transporte das mochilas escolares; orientações sobre carga do material de uso diário exigido pela escola e orientações 
para aquisição de materiais mais leves. Na palestra destinada às crianças, além destes conteúdos, foram também realizadas vivências práticas. Após a intervenção, as crianças receberam reforços educativos durante três meses, com frequência de 1 sessão de 60 minutos por mês. Nesses encontros mensais, as orientações práticas foram reforçadas na sala de aula. Do mesmo modo, foi enviado um folheto com conteúdo resumido da palestra para os pais e/ou responsáveis, e foram fixados nas salas de aula cartazes para servir de reforço aos professores e aos próprios alunos. Os escolares foram avaliados antes do início das palestras e após o fim dos reforços. Estes pesquisadores verificaram que esta intervenção promoveu mudanças significativas no transporte de mochilas, principalmente com relação à diminuição da quantidade de carga (relativa e absoluta) transportada. Especificamente, os resultados mostraram um aumento significativo do número de alunos que utilizavam mochila de duas alças, sendo esta significativamente transportada por mais alunos de forma bilateral sobre os ombros.

Rebolho, Casarotto e Amado (2009) objetivaram verificar a contribuição de duas estratégias educacionais na retenção de informações sobre hábitos posturais adequados. Os escolares foram submetidos a dois diferentes programas de educação postural: a) história em quadrinhos e b) experiência prática. Foram ministrados aos dois grupos, os mesmos conteúdos sobre hábitos posturais, porém por meio de diferentes estratégias de ensino-aprendizagem. Os escolares participaram de quatro encontros sendo desenvolvidos os seguintes conteúdos: anatomia e biomecânica do sistema musculoesquelético e articular, e hábitos posturais adequados. As avaliações, realizadas após seis meses do término da intervenção por meio de um questionário de conhecimentos sobre hábitos posturais adequados, demonstraram que as duas estratégias de ensino utilizadas mostraram-se efetivas no sentido de ensinar e fixar conceitos dos hábitos posturais.

Candotti et al (2009) objetivaram verificar a contribuição de uma Escola Postural com metodologia adaptada para crianças em proporcionar mudanças nos hábitos de postura nas AVD's e ajustes 
posturais estáticos da cabeça e ombros em busca de uma postura adequada, e proporcionar informações acerca da coluna vertebral. Nos períodos avaliativos foram realizadas as seguintes avaliações com ambos os grupos: (a) Avaliação da Postura Estática, avaliada no posturógrafo, visando avaliar a postura estática nos planos frontal e sagital, bem como observar possíveis desvios posturais do avaliado; (b) Avaliação da Postura Dinâmica, avaliada a partir de um circuito de AVD's, proposto por Rocha e Souza (1999) com registros em vídeo; e (c) Questionário informativo sobre os conhecimentos teóricos, o qual objetivou verificar o conhecimento teórico sobre a coluna vertebral. O Programa de Escola Postural desenvolvido neste estudo (CANDOTTI et al, 2009) para crianças entre oito e onze anos de idade, foi adaptado de Souza (1996). Os conteúdos desenvolvidos foram: estrutura e funções das estruturas da coluna vertebral, objetivos da Escola Postural, postura adequada nas AVD's. Os resultados demonstraram que o Programa de Escola Postural foi eficaz no aprendizado da execução das AVD's e dos conhecimentos teóricos sobre a coluna vertebral logo após a finalização do programa, não sendo eficaz no realinhamento da postura estática.

Benini e Karolczak (2010) objetivaram analisar os benefícios de um programa de educação postural em escolares de uma escola de ensino fundamental. O programa educativo consistiu em uma única sessão educativa aos escolares e professores, sendo desenvolvidos os seguintes conteúdos: anatomia e as lesões da coluna vertebral, e informações sobre modo de transporte e excesso de peso da mochila escolar, e sobre as posturas adequadas nas AVD's. Os escolares foram avaliados quatro semanas após a intervenção por meio de um questionário sobre hábitos posturais na escola e nas AVD's. Os pesquisadores, a partir dos resultados deste estudo, concluíram que apenas uma sessão de educação postural para alunos promoveu o conhecimento de hábitos posturais saudáveis e a modificação de algumas posturas cotidianas.

Em estudo também publicado em 2010, Candotti et al (2010) desenvolveram uma pesquisa similar à publicada em 2009, descrita anteriormente (CANDOTTI et al, 2009), com pubescentes. Os 
pesquisadores objetivaram verificar a contribuição de um programa de escola de postura em proporcionar mudanças nos hábitos posturais. Nos períodos de pré e pós-experimento, além dos procedimentos de avaliação realizadas por Candotti et al (2009), foi utilizado também um Questionário de Dor. A Escola de Postura proposta neste estudo é idêntica à proposta por Candotti et al (2009). Os resultados desse estudo sugerem que a Escola de Postura Adaptada para pubescentes teve um efeito positivo quanto: (a) ao realinhamento da postura estática, (b) ao conhecimento teórico sobre o funcionamento da coluna vertebral e suas estruturas, (c) à postura dinâmica na execução das atividades do dia a dia do pubescente, e (d) à diminuição da dor nas costas.

A mais recente publicação encontrada foi desenvolvida por Candotti et al (2011). Este estudo teve como objetivo avaliar os efeitos de um Programa de Educação Postural para as crianças e adolescentes oito meses após o seu término. Este estudo foi um follow-up de dois estudos realizados anteriormente pelos mesmos autores (CANDOTTI et al, 2009; CANDOTTI et al, 2010). Utilizando os mesmos procedimentos de avaliação anteriores (CANDOTTI et al, 2009) os autores buscaram verificar se o efeito apresentado imediatamente após o término dos Programas de Educação Postural era mantido após oito meses sem qualquer intervenção. Os resultados encontrados sugerem que os efeitos positivos resultantes do Programa de Educação Postural não foram estendidos ao período de follow-up. Portanto, os participantes do Programa de Educação Postural, oito meses após seu término demonstraram: (a) não conhecer e não saber identificar a coluna vertebral, suas partes e funções; e (b) não manter as curvaturas naturais da coluna vertebral, seja durante a postura estática ou durante as AVD's. Estes resultados sugerem que os conteúdos teóricos e o conhecimento da importância da boa postura durante as AVD's não foram efetivamente incorporados aos hábitos das crianças e adolescentes, sinalizando a necessidade de rever a metodologia de ensino utilizada e o tempo ou carga horária utilizada para desenvolvimento das atividades através de novas pesquisas. 


\section{Apontamentos finais}

A partir da análise dos objetivos das nove publicações verificase que estas apresentam objetivos similares, visto que pretendem avaliar a contribuição de programas de ensino para a aquisição de hábitos posturais adequados. No entanto, diferem no que tange a avaliação, pois algumas publicações enfatizam a contribuição do programa a partir da melhora dos conhecimentos e outras a partir da execução (prática) das AVD's. A estrutura dos programas variou desde um único encontro até vinte encontros; de aula direcionada exclusivamente para escolares à aula para escolares, pais, corpo docente e direção da escola. No que diz respeito às metodologias de ensino destes programas, de forma geral, as publicações apresentam métodos de ensino bastante distintos entre si. As metodologias de ensino contemplaram desde aulas expositivas à utilização de diversos recursos pedagógicos como filmes, pôsteres, transparências e vivências corporais. Quanto aos conteúdos abordados nestes programas de ensino, alguns tópicos em comum foram encontrados, tais como: anatomia e biomecânica da coluna vertebral, e postura adequada nas AVD's, o que é também observado nos programas direcionados a população de adultos. Entretanto, cada estudo apresenta particularidades individuais, como o ensino detalhado sobre os desvios posturais, sobre as lesões da coluna vertebral, metodologias baseadas em história em quadrinhos e vivências corporais. Isto demonstra que os pesquisadores não seguem uma única metodologia de ensino, pois utilizam estratégias pedagógicas diversificadas para o ensino da postura corporal.

Como instrumento avaliativo, utilizado para comparar as avaliações antes e após as intervenções, predomina a utilização de questionários, seja para avaliar o conhecimento teórico, a postura corporal nas AVD's, e/ou a dor nas costas. Dentre as nove publicações, oito utilizaram o questionário como instrumento avaliativo, e em quatro destas, o questionário foi o único instrumento avaliativo (Quadro 1). Referente a isto, Andreotti e Okuma (1999) relatam que existe a necessidade de serem utilizados conjuntamente aos questionários, testes dinâmicos padronizados e validados para 
avaliação da postura dinâmica, elevando a qualidade da avaliação, visto que os questionários em geral não são capazes de mensurar a incorporação do conhecimento teórico para a prática, ou seja, a transferência do conhecimento para a execução do movimento, fato este facilmente obtido por meio da filmagem da postura dinâmica (SPENCE; JENSEN; SHEPARD, 1984). Possivelmente, por este motivo, o grupo de pesquisadores do Rio Grande do Sul (RITTER, 2003; CANDOTTI et al, 2009; CANDOTTI et al, 2010; CANDOTTI et al, 2011) e de São Paulo (FERNANDES; CASAROTTO; JOÃO, 2008), na busca por avaliações de maior qualidade, tem utilizado, conjuntamente aos questionários, testes dinâmicos padronizados para avaliação da postura dinâmica.

Fernandes, Casarotto e João (2008) utilizaram a filmagem com o objetivo apenas de verificar o modelo e o modo de transporte da mochila escolar dos avaliados. Já Candotti et al (2009; 2010; 2011) e Ritter (2003) utilizaram como instrumento avaliativo a "Observação das Atividades de Vida Diária através de vídeo", instrumento este proposto e validado por Rocha e Souza (1999). Este instrumento, com o objetivo de avaliar a postura durante a execução de seis AVD's, foi submetido à validação de conteúdo por três especialistas em postura corporal, e após, à consistência interna e fidedignidade, sendo verificado um elevado coeficiente alfa e elevados valores de correlação, respectivamente. Embora muito acessível e de fácil aplicação, este instrumento foi validado com uma amostra composta por trabalhadores adultos, e por isso não avaliou AVD's comuns no contexto escolar, como, por exemplo, transportar mochila e utilizar o computador. Não obstante, uma vez que o instrumento proposto por Rocha e Souza (1999) não foi concebido para o ambiente escolar, entende-se que ainda existe a necessidade do desenvolvimento e validação de instrumentos específicos para avaliar a postura corporal nas AVD's rotineiras dos escolares, para que se possa avaliar de forma mais eficaz esta população.

Os programas de ensino, avaliados por meio de questionário, filmagem da execução das AVD's, assim como por testes de 
amplitude de movimento e de avaliação da postura estática, demonstraram resultados similares. De forma geral, os programas de ensino foram eficazes na melhora imediata dos conhecimentos teóricos sobre os conteúdos ensinados, e na melhora da execução das AVD's, ou seja, demonstraram que os escolares foram capazes de assimilar os conhecimentos teóricos e, em algumas situações, de levá-los para a prática. Ainda, o peso da mochila escolar, avaliado em um único estudo, diminuiu após a realização das sessões educativas (FERNANDES; CASAROTTO; JOÃO, 2008). No entanto, a melhora da amplitude de movimento articular e a contribuição das intervenções no realinhamento da postura estática ainda não foram alcançadas, embora estes tipos de resultados não sejam objetivos específicos das Escolas Posturais.

Como se pode observar, de forma geral, os resultados da presente revisão sistemática demonstraram a contribuição dos programas de ensino na melhora imediata dos conhecimentos teóricos e na capacidade dos escolares levarem este conhecimento à prática, quando avaliada logo após o término dos programas. Estes dados corroboram com estudos de intervenção realizados para adultos (UES; MORAES, 2003, FERREIRA; NAVEGA, 2010; TOBO et al, 2010; BORGES et al, 2011) e são encorajadores para que mais experiências desta natureza sejam desenvolvidas e integradas ao programa escolar. $\mathrm{Na}$ análise dos resultados de cada estudo, um aspecto que merece destaque, diz respeito ao delineamento metodológico. Segundo Thomas, Nelson e Silverman (2012), os estudos experimentais, compostos por grupos experimental e controle, apresentam maior validade interna quando comparados aos estudos compostos por apenas um grupo experimental, visto que existe maior garantia e possibilidade de afirmar que os resultados obtidos se devem de fato à intervenção. Sugere-se que novas pesquisas de escolas posturais com escolares concentrem esforços para agregar um grupo controle no seu delineamento metodológico, possibilitando uma avaliação das repercussões de uma intervenção específica sobre a postura.

No entanto, embora se verifique os efeitos positivos das Escolas 
Posturais e programas de ensino similares, estudos que visem identificar se estas mudanças são permanentes ainda são escassos. Entre as nove publicações encontradas realizadas com a população de escolares no Brasil, apenas uma, a mais recente (CANDOTTI et al, 2011), objetivou avaliar se os conhecimentos adquiridos permaneciam após um longo período sem intervenção. Os autores concluíram que os aprendizados adquiridos após a intervenção não se mantiveram por um período de oito meses demonstrando que um intervalo desta magnitude, sem orientação e reforço das novas aprendizagens, provavelmente deve ter afetado negativamente os efeitos do programa em longo prazo. Segundo Weineck (2005) movimentos incorporados se perdem do ponto de vista mecânico e fisiológico quando não são renovados de tempo em tempo, o que justifica o retrocesso dos conhecimentos adquiridos com a Escola Postural. A incorporação de novos conhecimentos está associada a uma metodologia que sistematize e dê significado para que os mesmos façam parte do cotidiano dos escolares.

Um aspecto relevante na aprendizagem e na incorporação dos conhecimentos teóricos é a motivação dos escolares na participação dos programas de ensino, pois a motivação é dinamizadora da aprendizagem, conduzindo os seres humanos à ação ou à inércia, ou seja, é o motivo pelo qual se escolhe fazer ou manter algo. Assim, os autores (CANDOTTI et al, 2011) acreditam que a motivação foi fundamental para a realização do PEP, pois a mesma é decisiva no processo de aprendizagem, sendo responsável pela inércia ou pela ação em qualquer atividade. Cardon, Clercq e Bourdeaudhuij (2002), desenvolveram uma Escola Postural de seis aulas para 347 escolares da quarta e quinta séries da Bélgica, encontrando resultados positivos em relação ao conhecimento teórico e à realização das AVD's logo após a finalização da intervenção e no follow-up de um ano, resultados que demonstram a possibilidade de incorporação de conhecimentos e de hábitos posturais adequados por escolares.

Candotti, Rohr e Noll (2011) identificaram, junto a professores de Educação Física do Ensino Fundamental II da cidade de Montenegro, que $90,9 \%$ dos professores consideram importante 
ensinar e praticar a educação postural com os alunos, mas que apenas 13,6\% trabalhavam este conteúdo específico em suas aulas. Dos 22 professores que participaram dessa pesquisa, 50\% não haviam cursado disciplinas que contemplassem conteúdos de educação postural na graduação. Para que mudanças ocorram no campo da atuação profissional, é necessário que os cursos de graduação invistam em disciplinas focadas na Educação Postural. Nesse sentido, a inclusão de uma disciplina de educação postural na formação dos professores de Educação Física estimularia a incorporação da educação postural no planejamento das aulas, cumprindo-se com conteúdo obrigatório no ensino básico previsto nos Parâmetros Curriculares de Educação Física (1997).

\section{Perspectivas}

Buscando minimizar os efeitos tardios negativos e para fomentar a utilização da Escola Postural, uma sugestão seria a implantação de "reforços de aprendizagem" periódicos, ou seja, que após a participação destes programas de ensino, os participantes fossem convidados a retornar em períodos inferiores a oito meses para vivenciar, por exemplo, em um único encontro, as experiências corporais e posturais experimentadas na Escola Postural.

Diante destes resultados, acredita-se ser importante continuar a implantar e estudar os efeitos de uma Escola Postural, com avaliações em longo prazo, para averiguar a permanência dos conteúdos adquiridos. Assim, torna-se interessante que novos programas de Escola Postural sejam realizados com crianças e adolescentes, porém é necessário que, após o término destes programas, houvessem reforços periódicos por meio de atividades mensais como palestras e/ou vivências posturais. Especula-se que estas atividades possivelmente garantiriam o aprendizado e assimilação dos conteúdos da Escola Postural, sendo incorporados aos hábitos dos escolares. Entretanto, novas investigações científicas são necessárias para comprovar ou refutar esta hipótese.

Em suma, entende-se fundamental que se inicie, logo na 
infância, uma orientação postural adequada, alertando do perigo das atitudes corporais inadequadas durante a execução das AVD's e mostrando as posturas corretas a serem realizadas diariamente. Cardon et al (2007) e Candotti, Roth e Noll (2012) relatam a importância dos programas de orientação e prevenção serem iniciados já nos primeiros anos do Ensino Fundamental, pois deste modo grande porcentagem da população poderia ser educada, visto que, por lei, toda criança deve estar regularmente matriculada no ensino básico. Além disto, o início destes programas nos primeiros anos do ensino básico possibilita a realização de vários reforços de aprendizagem (CARDON et al, 2007, CANDOTTI; ROTH; NOLL, 2012), o que proporciona aos jovens a possibilidade de aprender a estabelecer padrões adequados de movimento sem ter que quebrar padrões ineficientes e hábitos inadequados. 


\begin{abstract}
Back school: systematic review of programs designed for schoolchildren in Brazil

Abstract: This study aims to present and discuss the Back School programs developed for students in Brazil. It was used a methodology for systematic review of scientific articles in several data bases and in the Bank of Thesis and Dissertations of the Capes. From nine publications included in this study it was concluded that Back School programs contribute to the acquisition of the theoretical knowledge and postural habits healthy during the execution of activities of daily living. However, studies aimed to identify whether the knowledge and the postural habits acquired are kept in the course of time are still scarce.
\end{abstract}

Keywords: Posture. Spine. Health Education. Students. Brazil.

\section{Educación postural: revisión sistemática de programas educativos desarrollados en Brasil Resumén: Este estudio tiene como objetivo presentar y discutir los programas de Escuela Postural desarrollados para los estudiantes de lo Brasil. Se utilizó una metodología sistemática de artículos científicos en bases de datos y Banco Tesis e Disertaciones Capes. A partir de las nueve publicaciones incluidas en este estudio se deduce que los programas de Escuela Postural son eficaces para mejorar los conocimientos teóricos y para mejorar el desempeño de las actividades de la vida diaria. Sin embargo, los estudios encaminados a identificar los conocimientos y hábitos posturales adquiridos se conservan en el transcurso del tiempo escasos. \\ Palabras clave: Postura. Columna Vertebral. Educación en Salud. Estudiantes. Brasil.}

\section{REFERÊNCIAS}

ANDRADE, SC; ARAÚJO, AG, VILAR, MJ. Escola de coluna: Revisão Histórica e sua aplicação na lombalgia crônica. Revista Brasileira de Reumatologia, São Paulo, v. 4, p. 224-228, 2005.

ANDREOTTI, RA; OKUMA, SS. Validação de uma bateria de testes de atividades da vida diária para idosos fisicamente independentes. Revista Paulista de Educação Física, São Paulo, v. 1, p. 46-66, 1999.

AUVINEN, JP et al. Is insufficient quantity and quality of sleep a risk factor for neck, shoulder and low back pain? A longitudinal study among adolescents. European Spine Journal, Zurique, v. 19, p. 641-649, 2010. 
BENINI, J; KAROLCZAK, APB. Benefícios de um programa de educação postural para alunos de uma escola municipal de Garibaldi, RS. Fisioterapia e Pesquisa, São Paulo, v. 4, p. 346-351, 2010.

BORGES RG et al. Efeitos da participação em um Grupo de Coluna sobre as dores musculoesqueléticas, qualidade de vida e funcionalidade dos usuários de uma Unidade Básica de Saúde de Porto Alegre. Motriz, Rio Claro, v. 17, n. 4, p. 719727, 2011.

CANDOTTI, CT et al. Efeitos de um programa de educação postural para crianças e adolescentes após oito meses do seu término. Revista Paulista de Pediatria, São Paulo, v. 29, n. 4, p. 577-583, 2011.

. Escola de postura: uma metodologia adaptada aos pubescentes. Revista Mackenzie de Educação Física e Esporte, São Paulo, v. 2, p. 91-100, 2010.

. Escola postural: uma metodologia adaptada para crianças. Revista Arquivos em Movimento, Rio de Janeiro, v. 2, p. 34-49, 2009.

CANDOTTI, CT; NOLL, M; CRUZ, M. Prevalência de dor lombar e os desequilíbrios musculares em manicures. Revista Arquivos em Movimento, Rio de Janeiro, v. 1, p. 125-140, 2010.

CANDOTTI, CT; ROHR, J; NOLL M. A Educação Postural como conteúdo curricular da Educação Física no Ensino Fundamental II nas escolas da Cidade de Montenegro/ RS. Movimento, Porto Alegre, v. 17, n. 3, p. 57-77, 2011.

CANDOTTI, CT; ROTH, E; NOLL, M. Evaluation of weight and mode of transport of student in school of education. Revista Paulista de Pediatria, São Paulo, v. 30, n. 1, p. 100-6, 2012.

CARDON, G et alBack education in elementary schoolchildren: the effects of adding a physical activity promotion program to a back care program. European Spine Journal, Zurique, v. 16, p. 125-133, 2007.

CARDON, G; CLERCQ, D; BOURDEAUDHUIJ, I. Back education efficacy in elementary schoolchildren: a 1-year follow-up study. Spine, Philadelphia, v. 3, p. 299-305, 2002.

. Effects of back care education in elementary schoolchildren. Acta Paediatric, Estocolmo, v. 89, p. 1010-1017, 2000.

CHUNG, TM. Escola de Coluna - Experiência do Hospital das Clínicas da Universidade de São Paulo. Acta Fisiátrica, São Paulo, v. 2, p. 13-17, 1996.

DETSCH C et al. P. Prevalência de alterações posturais em escolares do ensino médio em uma cidade no Sul do Brasil. Revista Panamericana de Salud Publica, Washington, v.4, p.231-238, 2007.

DETSCH, C; CANDOTTI, CT. A incidência de desvios posturais em meninas de 6 a 17 anos da cidade de Novo Hamburgo. Movimento, Porto Alegre, v. 8, p. 43-56, 2001. 
FERNANDES, SMS; CASAROTTO, RA; JOÃO, SMA. Effects of educational sessions on school backpack use among elementary school students. Revista Brasileira de Fisioterapia, São Carlos, v. 6, p. 447-453, 2008.

FERREIRA, MS, NAVEGA, MT. Efeitos de um programa de orientação para adultos com lombalgia. Acta Ortopédica Brasileira, São Paulo, v. 3, p. 127-131, 2010.

FORSSEL, MZ. The Swedish Back School. Physiotherapy, Kidlington, v. 66, n. 4, p. $112-14,1980$.

FORSSEL, MZ. The Back School. Spine, Philadelphia, v. 6 p. 104-06, 1981.

GALVÃO, CM; SAWADA, NO; TREVIZAN, MA. Revisão sistemática: recurso que proporciona a incorporação das evidências na prática da enfermagem. Revista Latino-americana de Enfermagem, Ribeirão Preto, v. 12, n. 3, p. 549-56, 2004.

KARAHAN, A; BAYRAKTAR, N. Determination of the usage of body mechanics in clinical settings and the occurrence of low back pain in nurses. International Journal Nursing Studies, Londres, v. 41, p. 67-75, 2004.

KELLIS, E; EMMANOUILIDOU, M. The effects of age and gender on the weight and use of schoolbags. Pediatric Physical Therapy, Virginia, v. 22, p. 17-25, 2010.

KNOPLICH, J. Qual deveria ser o nome da Back School Brasileira? Revista Brasileira de Reumatologia, São Paulo, v. 1, p. 80-81, 2006.

LIMON, S; VALINSKY, LJ; SHALOM, YB. Risk factors for low back pain in the elementary school environment. Spine, Philadelphia, v. 6, p. 697-702, 2004.

MÉNDEZ, FJ; GÓMEZ-CONESA, A. Postural hygiene program to prevent low back pain. Spine, Philadelphia, v. 11, p. 1280-1286, 2010.

NETO, F. Avaliação Postural em Escolares de 1aa $4^{\text {a }}$ Série do 10 Grau. Revista Brasileira Ciência e Movimento, Taguatinga, v. 2, p. 7-11, 1991.

PAANANEN, MV et al. Risk factors for persistence of multiple musculoskeletal pains in adolescence: A 2-year follow-up study. European Journal of Pain, Chichester, v. 10, p. 1026-1032, 2010.

PEZZAN, PAO et al. Postural Assessment of Lumbar Lordosis and Pelvic Alignment Angles in Adolescent Users and Nonusers of High-Heeled Shoes. Journal Manipulative and Physiological Therapeutic, Nova lorque, v. 34, n. 9, p. 614621, 2011

REBOLHO, MCT; CASAROTTO, RA; AMADO, SM. Estratégias para ensino de hábitos posturais em crianças: história em quadrinhos versus experiência prática. Fisioterapia e Pesquisa, São Paulo, v. 1, p. 46-51, 2009.

RITTER, AL. Programa Postural para escolares do Ensino Fundamental. Dissertação (Mestrado) - Escola de Educação Física, Programa de Pós Graduação 
de Ciências do Movimento Humano, Universidade Federal do Rio Grande do Sul, Porto Alegre, 2003.

ROBERTSON, HC; LEE, VC. Effects of back care lessons on sitting and lifting by primary students. Australian Physiotherapy, Hawthorn, v. 4, p. 245-248, 1990.

ROCHA, A; SOUZA, JL. Observação das atividades de vida diária através de vídeo. Movimento, Porto Alegre, v. 11, p. 16-22, 1999.

SANTOS, Cl et al. Ocorrência de desvios posturais em escolares do ensino público fundamental de Jaguariúna, São Paulo. Revista Paulista de Pediatria, São Paulo, v. 1 , p. $74-80,2009$.

SANTOS, SG. Educação postural mediante um trabalho teórico. Revista Brasileira de Atividade Física e Saúde, Florianópolis, v. 2, p. 32-42, 1998.

SHEHAB, DK; JARALLAH, KF. Nonspecific low-back pain in Kuwaiti children and adolescents: associated factors. Journal of Adolescent Health, São Francisco, v. 36, p. 32-35, 2005.

SIIVOLA, S et al. Predictive Factors for Neck and Shoulder Pain: A longitudinal Study in Young Adults. Spine, Philadelphia, v. 15, p. 1662-1669, 2004.

SILVA, VRL; MENEZES, AMB; NOAL, RB. Sintomas articulares crônicos em adultos de Pelotas, Rio Grande do Sul, Brasil: prevalência e determinantes. Cadernos de Saúde Pública, Rio de Janeiro, v. 25, p. 2571-2582, 2009.

SKOFFER, B. Low Back Pain in 15 to 16 year old children in relation to school furniture and carrying of the school bag. Spine, Philadelphia, v. 24, p. 713-717, 2007.

SOUZA, AS et al. Efeitos da escola de postura em indivíduos com sintomas de lombalgia crônica. ConSientiae Saúde, São Paulo, v. 3, p. 497-503, 2010.

SOUZA, JL. Efeitos de uma Escola Postural para indivíduos com dores nas costas. Movimento, Porto Alegre, v. 5, p. 56-71, 1996.

SOUZA, JL; VIEIRA, A. Escola Postural: um caminho para o conhecimento de si e o bem-estar corporal. Movimento, Porto Alegre, v. 3, p. 101-122, 2003.

SPENCE, SM; JENSEN, GM; SHEPARD, KF. Comparison of methods of teaching children proper lifting techniques. Physical Therapy, Virginia, v. 64, n. 7, p. 10551066, 1984.

THOMAS JÚNIOR, NELSON JK; SILVERMAN, S. Métodos de pesquisa em atividade física. Porto Alegre: Artmed, 2012.

TOBO, A et al. Estudo do tratamento da lombalgia crônica por meio da Escola de Postura. Acta Fisiátrica, São Paulo, v. 3, p. 112-116, 2010. 
TREVELYAN, FC; LEGG, SJ. Back pain in school children: where to from here? Applied Ergonomics, Madison, v. 37, p. 45-54, 2006.

UES, ME; MORAES, JP. Escola postural para a Terceira idade. Praxisterapia, Local, v. 8, p. 53-64, 2003.

VANDERTHOMMEN, M et al. Le comportement gestual du patient lobalgique fréquentant une école du dos: analyse préliminaire dún test d'évaluation. Annales de Réadaption et de Médecine Physique, Cidade do México, v. 42, p. 485-492, 1999.

VIEIRA, A, SOUZA, JL. Concepções de boa postura dos participantes da Escola Postural da ESEF/UFRGS. Movimento, Porto Alegre, v. 1, p. 9-20, 2002.

WEINECK, J. Biologia do esporte. 7. ed. São Paulo: Manole; 2005.

WOMERSLEY, L; MAY, S. Sitting posture of subjects with postural backache. Journal of Manipulative and Physiological Therapeutics, Arlington, v. 29, n. 3, p. 213218, 2006.

ZAPATER, AR et al. Postura sentada: a eficácia de um programa de educação para escolares. Ciência e Saúde Coletiva, Rio de Janeiro, v. 9, n. 1, p. 191-199, 2004.

Endereço para correspondência:

Matias Noll

Rua Guilherme Brust 742,

Bairro Languiru, Teutônia, RS

Cep 95890-000

Recebido em: 21.11.2011

Aprovado em: 04.09.2012 